\title{
TEMPERATURE AND
}

\section{BEHAVIOURAL RESPONSES INDUCED IN THE UNANAESTHETIZED CAT BY THE CENTRAL ADMINIS- TRATION OF RX72601, A NEW ANTICHOLINESTERASE}

\author{
G. METCALF, R.D. MYERS \& P.C. REDGRAVE \\ Laboratory of Neuropsychology, Purdue University, West Lafayette, Indiana 47907, U.S.A.
}

1 The central effects produced by a new anticholinesterase RX72601 injected into the cerebral ventricles or perfused within circumscribed forebrain structures have been investigated in the unanaesthetized cat.

2 When injected into the cerebral ventricles in doses of 1-10 $\mathrm{g}, \mathrm{RX} 72601$ produced a dose-related hyperthermia together with behavioural and autonomic responses.

3 Experiments designed to localize the site or sites of these actions demonstrated that hyperthermia could be produced from several diencephalic sites but that the other effects were not normally produced from these same sites.

\section{Introduction}

Two distinct types of evidence support the concept that a cholinergic pathway within the hypothalamus mediates heat production (Myers, 1971a). Firstly, acetylcholine (ACh) and cholinomimetic substances injected into the ventricles or directly into the brain substance produce a rise in body temperature in a number of species (for review, see Myers, 1974). Secondly, on perfusion of discrete hypothalamic sites in the monkey with push-pull cannulae, it has been shown that the release of ACh from such sites is increased mainly by cooling and suppressed by warming the primate's body (Myers \& Waller, 1973).

Attempts to identify the type of cholinoceptor involved in thermoregulatory processes have also proceeded along two separate avenues. By means of micro-injection techniques in primates, Myers and his co-workers have mapped out areas of the diencephalon most sensitive to cholinergic stimulation using carbachol (Myers \& Yaksh, 1969) and later nicotine (Hall \& Myers, 1971). Briefly, these workers found that whilst both drugs produced hyperthermia when injected into the posterior hypothalamus only carbachol did so when injected into the anterior hypothalamus and preoptic area. A similar study was undertaken in the cat by Rudy \& Wolf (1972) who also used carbachol as their cholinomimetic agent. They too found that this substance was capable of producing hyperthermia in both the anterior and posterior regions of the hypothalamus. Although the drug-induced changes in body temperature reported by all of these workers depend on the site of injection and the dose administered, it is not clear whether the receptors involved can be clearly designated muscarinic or nicotinic as is the custom for peripheral cholinoceptors.

The second approach was pharmacological rather than anatomical in nature. Hall (1972) induced hyperthermia in cats by the intraventricular injection of carbachol. Similar injections of nicotine produced hypothermia. The carbachol-induced hyperthermia was abolished by atropine but not by hexamethonium or mecamylamine. Conversely, the nicotine hypothermia was blocked by hexamethonium or mecamylamine but not by atropine. Although these findings support the view that cholinergic hyperthermia results from stimulation of 'muscarinic' receptors whilst cholinergic hypothermia is produced as the result of stimulating 'nicotinic' receptors, nicotine injected directly into the caudal hypothalamus of the monkey produces a rise in temperature (Hall \& Myers, 1972).

All the experiments described so far have sought to identify and characterize responses achieved by the direct introduction of cholinomimetic agonists into the central nervous system (CNS). An alternative approach is to observe the effects produced when the breakdown of endogenous $\mathrm{ACh}$ is prevented at selected CNS sites by the prior administration of an anticholinesterase to those sites. This paper reports the effects observed when the anticholinesterase RX72601, i.e. cis-2-(3-hydroxy-phenyl)-1pyrrolidino cyclohexane methobromide (Dettmar, 
Lewis, Metcalf, Readhead, Smith, Gillett, Hedges \& Richens, 1974a; Dettmar, Metcalf, Smith, Gillett, Hedges \& Richens, 1974b) was injected into the ventricles, or perfused at selected diencephalic sites, in the conscious cat. This compound is one of a series of anticholinesterases recently developed (Lewis, Readhead, Metcalf, Smith \& McNally, 1973) and was considered suitable for the present study because it has been shown to lack direct agonist activity at post-synaptic cholinoceptor sites (C.F.C. Smith, unpublished data).

\section{Methods}

Seventeen cats of either sex initially in the weight range of $2.6-3.5 \mathrm{~kg}$ were used. At least $48 \mathrm{~h}$ were allowed to elapse between subsequent experiments on individual animals.

\section{Surgical procedures}

Using the procedures described by Myers (1972) an array of either two or four guide tubes was implanted in each animal. Each guide tube consisted of an appropriate length of 17 gauge stainless steel tubing fitted with an indwelling stylet of the same length. The stereotaxic co-ordinates were chosen so that in animals implanted with two bilateral guide tubes the tips rested above the lateral ventricles, whilst in animals implanted with four guide tubes the bilateral arrangement allowed the tips to rest $4-6 \mathrm{~mm}$ above the rostral and caudal hypothalamus on each side of the brain. After the cannula array had been lowered to the appropriate position the guide tubes were anchored to the calvarium with cranioplast cement and stainless steel screws. A screw-top polyethylene pedestal was fastened over the array to protect the cannulae and prevent subsequent infection.

\section{Ventricular injections}

An injection was made into either lateral ventricle by means of a 20 gauge stainless steel needle which was lowered through the guide tube until contact with the ventricles was established (Myers, 1971b). At this time the solution to be injected passed rapidly into the ventricle by gravity flow. With the exception of one series of experiments, a standard volume of $0.2 \mathrm{ml}$ was used for all intraventricular injections.

\section{Push-pull perfusions}

To perfuse a discrete region of the diencephalon push-pull cannulae were used as described previously (Myers, 1970). The outer or pull cannula was constructed from 20 gauge stainless steel thin-wall tubing (Popper Co., New York, N.Y.) and the inner or push cannula from 27 gauge tubing. The push cannula was positioned so that its tip extended $0.8-1.0 \mathrm{~mm}$ beyond the end of the pull cannula. This concentric push-pull assembly was lowered through a guide tube to a depth controlled by a polyethylene spacer which fitted tightly over the shaft of the pull cannula. Both the push and pull cannulae were connected by polyethylene tubing to a $2.0 \mathrm{ml}$ calibrated glass syringe (Becton, Dickenson \& Co.) mounted on a 4-channel infusion-withdrawal pump (Harvard Apparatus Co.). All perfusions were made at the rate of $50 \mu 1 /$ minute.

\section{Injection solutions}

All solutions were prepared with glass distilled ion-exchanged water. An artificial cerebrospinal fluid (CSF) (Myers, 1971b) containing the chloride salts of the following cations (mM) $\mathrm{Na}^{+}$ $145.0, \mathrm{Ca}^{2+} 1.3, \mathrm{~K}^{+} 3.5$, and $\mathrm{Mg}^{2+} 1.0$ was used as the vehicle for drug injections. Both RX72601 and neostigmine (Sigma Ltd) were used as the bromide salts; doses refer to the salts. RX72601 (mol wt. 340) was supplied by Reckitt \& Colman Ltd, Hull. Each solution was rendered pyrogenfree by standard autoclaving procedures, and in addition was passed through a $0.22 \mu \mathrm{m}$ Swinnex Millipore filter just before injection. The push-pull cannulae, injection needles and polyethylene tubing were stored in $0.0013 \%$ benzalkonium chloride solution and flushed repeatedly with the solution to be injected just before use. Syringes and other glassware used were rendered pyrogen-free by autoclaving heating methods in an oven. Before an experiment began, a YSI 401 thermistor probe (Yellow Springs Instrument Co., Yellow Springs, Ohio) was inserted into the colon to a depth of $9-12 \mathrm{~cm}$ and held in place by surgical tape wrapped gently around the base of the tail. The temperature of each cat was recorded on a multi-channel potentiometric recorder for a baseline period of at least 1 hour. Animals were unrestrained throughout the experiments.

\section{Histology}

At the conclusion of a series of experiments ventricular injection sites were verified by the injection of $0.2 \mathrm{ml}$ bromothymol blue. The sites of push-pull perfusion were verified according to standard histological procedures. The cat was killed by an overdose of pentobarbitone sodium and $10 \%$ buffered, neutral formalin was perfused 


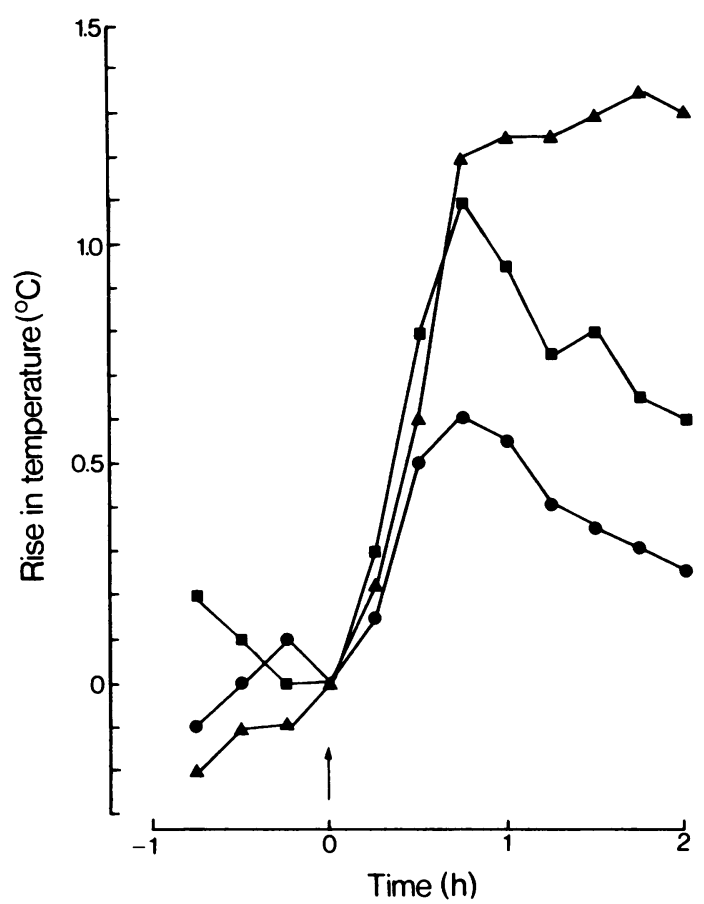

Figure 1 Hyperthermia induced in a cat by successive intraventricular injections (at arrow) of 1 (๑), 4 (a) and $10 \mu \mathrm{g} \mathrm{( \Delta )} \mathrm{RX72601,}$

through the thoracic aorta after the heart was clamped. The brain was washed and blocked; the sections were then cut at $100 \mu \mathrm{m}$ on a freezing microtome and stained for cells and fibres according to a procedure modified after Wolf (1971).

\section{Results}

\section{Intraventricular injections of $R X 72601$}

When injected into the lateral ventricle in a volume of $0.2 \mathrm{ml}, \mathrm{RX} 72601$ produced a dose-dependent rise in the cat's temperature over a ten-fold dose range. Doses of less than $1 \mu \mathrm{g}$ had very little effect on temperature. Doses greater than $20 \mu \mathrm{g}$ were not investigated. Figure 1 shows the differences in the hyperthermia produced in a single cat when progressively larger doses were injected in successive experiments. Although the onset of the hyperthermic responses was usually between 1 and $10 \mathrm{~min}$ after the injection, the duration of the fever produced was often difficult to assess, especially with larger doses, because of the interference encountered from a non-specific fever

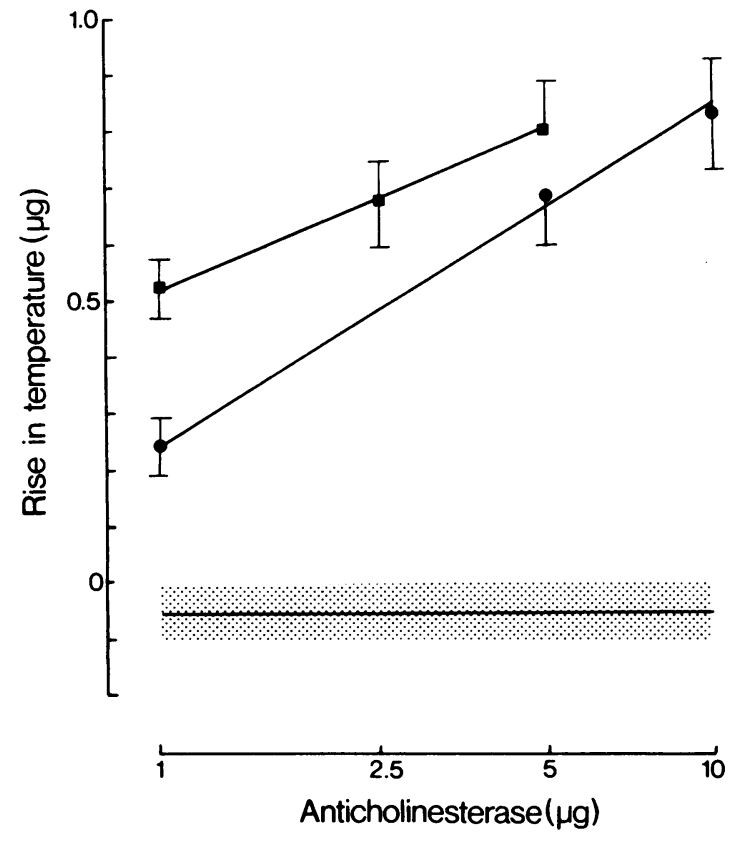

Figure 2 Hyperthermia induced in cats by the intraventricular injection of either $R \times 72601$ (๑) or neostigmine $(\square)$. Each point is the mean from 4-6 experiments. Vertical bars show s.e. mean. Hyperthermia was measured as the maximum rise in temperature produced within $1 \mathrm{~h}$ of injection. The shaded area represents the effect (mean \pm s.e.) produced by a similar injection of 5 ions.

of slow onset. This latter fever, which has been described by other workers (Feldberg, Myers \& Veale, 1970; Feldberg \& Saxena, 1970) and is thought to be due to an unknown pyrogenic factor, normally developed between 1 and $3 \mathrm{~h}$ after an injection. At such time the shorter duration hyperthermias produced by lower doses of RX72601 were completed, whilst the rises in temperatures produced by large doses of RX72601 were still in evidence. Figure 2 presents the relationship between the dose and maximum rise in temperature produced by RX72601 in the first hour after injection.

In a preliminary attempt to identify the anatomical region responsible for the drug-induced hyperthermia, $5 \mu \mathrm{g} \mathrm{RX72601}$ was injected into the lateral ventricles in three different volumes. Figure 3 shows that the greatest hyperthermia was produced when this dose was injected in a dose volume of $0.2 \mathrm{ml}$. Subsequent injections of bromothymol blue demonstrated that $0.05 \mathrm{ml}$ dye was contained within the lateral ventricle receiving the injection, that $0.1 \mathrm{ml}$ dye was distributed 


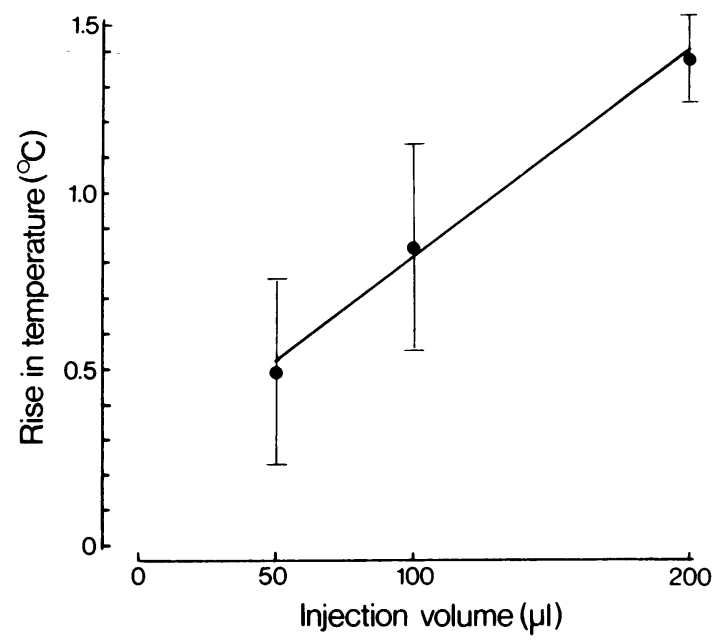

Figure 3 The effect of dose-volume on the hyperthermia produced by intraventricular injection

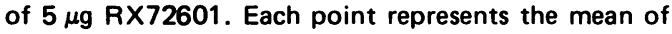
4 experiments. Vertical bars show s.e. mean.

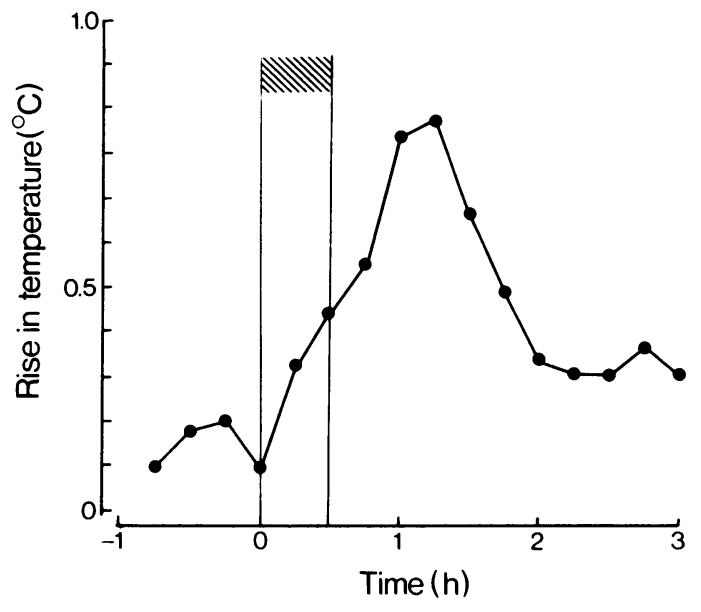

Figure 4 Change in body temperature of an unanaesthetized cat produced by a push-pull perfusion bilaterally of $R \times 72601(1.0 \mu \mathrm{g} / \mathrm{min})$ for 30 minutes. The sites in the dorsal hypothalamus were AP 11.0 as indicated in Figure 5 by the triangles ( $\Delta$ ). throughout the lateral ventricle and part of the third ventricle, whilst $0.2 \mathrm{ml}$ dye stained the whole of the lateral and third ventricles and sometimes extended caudally through the aqueduct into the fourth ventricle. Thus the main site of action for RX72601 appeared to be the structures which line the walls of the lateral and third ventricles.

Although the intensity of the symptoms observed varied on different occasions, the hyperthermia produced by the intraventricular injection was invariably accompanied by a behavioural syndrome. The symptoms produced included flattening of the ears and growling; no other responses of fear or aggression (Norton, 1969) were produced, however, and the animal could be handled without provoking an attack. Intense preening or scratching of the face and pinnae with the forepaws as if irritation was being experienced, was also observed. Higher doses $(10-20 \mu \mathrm{g})$ ordinarily produced pronounced shivering and sometimes mydriasis and nystagmus. The onset of the behavioural effects coincided with the onset of hyperthermia and they persisted intermittently for about 60-75 minutes. In contrast to the effect on body temperature, there appeared to be no diminution in the magnitude of the behavioural effects when RX72601 was injected in a smaller dose or volume. As with the body temperature effects, RX72601 had little discernible effect on behaviour at doses below $1 \mu \mathrm{g}$.

\section{Intraventricular injections of neostigmine}

To ascertain that the responses observed after the intraventricular injection of RX72601 were not independent of its anticholinesterase properties, the effect of another anticholinesterase, neostigmine, which is structurally unrelated to RX72601, was also tested. Figure 2 shows that a dose-related hyperthermia was also produced by neostigmine although the slope of the dose-response line is different from that of RX72601. Neostigmine also produced a similar behavioural syndrome to RX72601. In addition, the neostigmine-treated animals consistently resisted handling, although there was no actual attack upon the experimenter.

\section{Push-pull perfusion}

In order to localize more precisely the site of action for RX72601 a series of push-pull perfusions were performed at discrete tissue sites within the diencephalon and telencephalon. Perfusion with RX72601 in a concentration of $0.125 \mu \mathrm{g} / \mathrm{min}$ at any site failed to produce either hyperthermia or behavioural changes. When the concentration of RX72601 in the perfusing fluid was increased to $0.5-1.0 \mu \mathrm{g} / \mathrm{min}$, some sites were identified at which perfusion of this concentration for $30 \mathrm{~min}$ produced approximately $0.75^{\circ} \mathrm{C}$ rise in temperature. However, such hyperthermic responses appeared to be site specific for other sites 


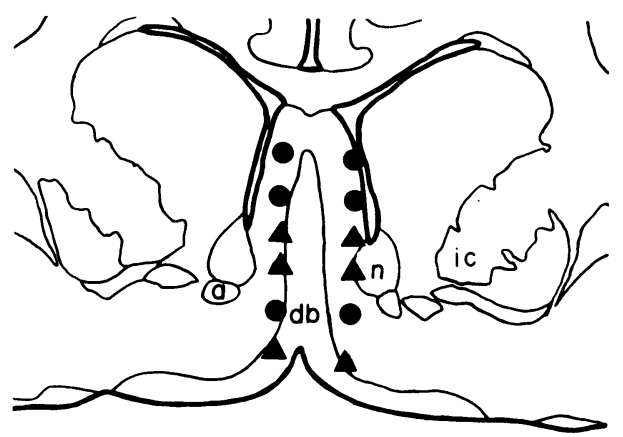

AP 16.0

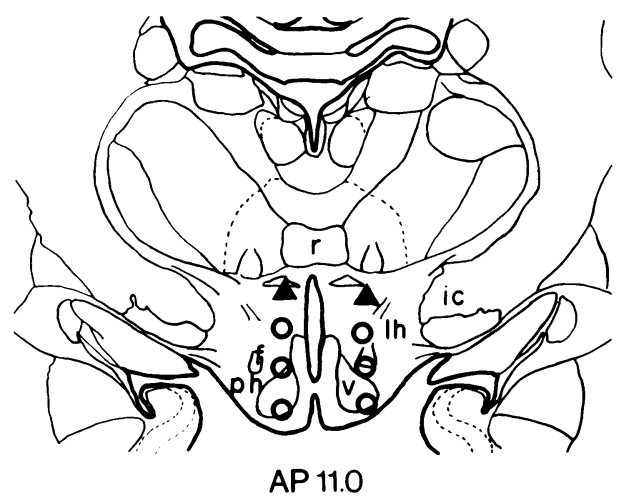

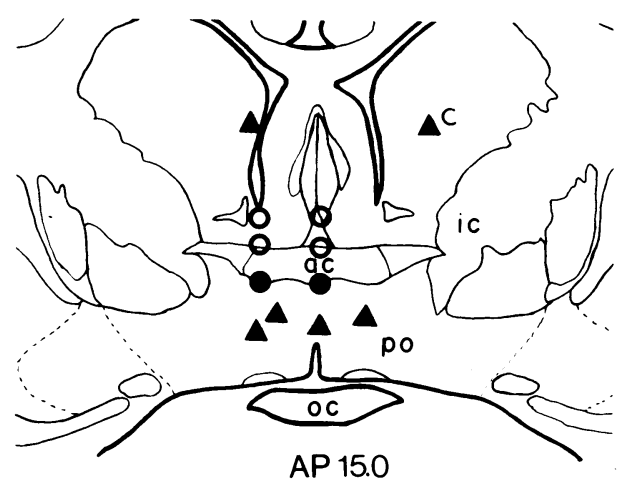

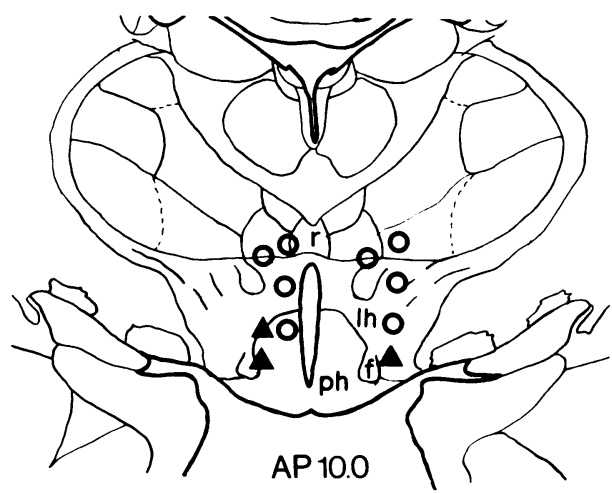

Figure 5 Representative frontal sections of cat brain illustrating the anatomical sites at which a push-pull perfusion of $R \times 72601$ produced a rise in temperature. ( $\Delta$ ) Indicates sites where push-pull perfusion of $1.0 \mu \mathrm{g} / \mathrm{min} \mathrm{R} \times 72601$ produced hyperthermia of $0.4^{\circ} \mathrm{C}$ or greater; (o) Indicates sites where doses of drug $0.125-0.5 \mu \mathrm{g} / \mathrm{min}$ were ineffective; $(\bullet)$ indicates sites where $1.0 \mu \mathrm{g} / \mathrm{min}$ was ineffective. Key to labelled structures; $a$, nucleus accumbens; ac, anterior commissure; c,caudate nucleus; db, diagonal band of Broca; $f$, fornix; ic, internal capsule; Ih, lateral hypothalamus; $n$, nucleus of anterior commissure; oc, optic chiasm; ph, posterior hypothalamus; po, pre-optic area; $r$, nucleus reuniens; $v$, ventromedial hypothalamus.

perfused with this concentration showed only minimal $\left(0.2-0.3^{\circ} \mathrm{C}\right)$ rises in body temperature. An example of a marked rise in temperature produced by perfusion of RX72601 at a site in the dorsal hypothalamus is illustrated in Figure 4.

Figure 5 indicates that the sites most sensitive to RX72601 were in the rostral hypothalamus, the ventral region of the septum, the caudate nucleus, the posterior region of the hypothalamus and at an area situated at the junction between the hypothalamus and the mid-brain at the level of AP10. However, in contrast to the intense behavioural syndrome observed after the intraventricular injection of $R \times 72601$, perfusion of the anticholinesterase at tissue sites failed to produce more than minimal behavioural effects.

\section{Discussion}

On the basis of previous experiments in which cholinomimetic agonists were injected directly into cerebral tissue, Myers $(1971 \mathrm{a}, 1974)$ has proposed that a cholinergic system located in the hypothalamus and mid-brain is involved in thermogenesis in the cat. The results obtained from the present experiments, in which the concentration of endogenous $\mathrm{ACh}$ in CNS structures is raised by inhibiting the enzyme responsible for its metabolic degradation, support this proposal. Such cholinergic thermogenesis has also been reported for other species. Both Myers \& Yaksh (1969) and Hall \& Myers (1971) have reported that hyperthermia is produced by 
micro-injection of cholinomimetic agonists into the hypothalamus of the monkey. Avery (1970) has observed a similar cholinergically-induced rise in temperature in rats, although in this species hypothermia has also been reported by other workers after CNS administration of cholinomimetic agents (for review see Myers, 1974).

Results obtained from both types of experiments designed to locate the anatomical site or sites at which $\mathrm{RX72601}$ acted to provoke hyperthermia support the concept that the cholinergic heat production pathway is diffuse in nature. Although sites in both the rostral and caudal hypothalamus appear to be critically involved in the hyperthermia, activity at the other sites lining the ventricles cannot be ruled out at this stage. Three further pieces of information are pertinent to this last point. Firstly, the compound is quaternary in nature and so will not cross biological membranes easily. This implies that such sites will be close to the ventricular walls. Secondly, when a cholinomimetic substance is injected directly into the caudate nucleus, hyperthermia has previously been reported (Connor, Rossi \& Baker, 1966). Thirdly, in the present experiments, the magnitude of hyperthermia produced by a push-pull perfusion of RX72601 at diencephalic sites never equalled the intensity of the response produced by an intraventricular injection implying, perhaps, that there is more than one site of action in the latter case. A more extensive study of push-pull perfusions or micro-injections will be necessary to define all of the anatomical sites at which RX72601 exerts its hyperthermic action.

Besides the effect on thermoregulation, an injection of RX72601 into the cerebral ventricles produced changes in the emotional behaviour of the cat. The animals crouched down with flattened ears and growled almost continuously for the first 50-60 min after the injection. Thereafter, the symptoms became intermittent and gradually decreased both in frequency and intensity. Intense face wiping, scratching or preening often accompanied the behavioural symptoms. A higher dose of RX72601 produced mydriasis and nystagmus. This duration of action corresponds to the duration of acetylcholinesterase inhibition previously measured in vivo (Dettmar et al., 1974b).

Much work has been done on cholinergically induced emotional behaviour since the original report (Myers, 1964) that micro-injections of carbachol or $\mathrm{ACh}$ into the cat's hypothalamus produced an affective defence reaction. The results obtained in the present study differ from some of the earlier results (for review see Myers, 1974) in two ways. Firstly, the spectrum of emotional behaviour observed during the present experiments is more limited than that previously reported. Secondly, such emotional behaviour as was observed occurred after intraventricular injection rather than push-pull perfusion at the selected diencephalic sites, as would perhaps have been expected from previous investigations.

Several explanations are possible for these differences. The doses of RX72601 used may have been too low to provoke the full behavioural syndrome, although a small number of subsequent experiments with higher doses do not confirm this suggestion. In addition, results from earlier work in both animals (Metcalf \& Dettmar, 1975; G. Metcalf, G. Shilcock \& M.H. Smith, unpublished observations) and man (Dettmar et al., 1974b) when applied to the present experiments suggest that the doses used would cause virtually complete inhibition of acetylcholinesterase in the restricted areas of drug application. Alternatively, most of the previous studies have used carbachol as the cholinomimetic agent; this drug is known to stimulate both muscarinic and nicotinic receptors in the periphery, and Myers (1974) has suggested that behavioural responses produced by this drug may result from stimulation of CNS receptors with muscarinic characteristics. By comparison, the results obtained previously with RX72601 suggest that this compound may be more active at nicotinic sites (Dettmar et al., 1974a, b). Thus the latter drug may exhibit a different spectrum of activity. The more complete aggressive syndrome observed after injection with neostigmine lends at least some credence to this latter explanation. Finally, it may be that the manipulation of the endogenous level of ACh offers an approach closer to physiological reality than the injection of synthetic cholinomimetic agonists resistant to normal metabolic degradation. Further experiments will be necessary to evaluate these possibilites.

This research was supported in part by grant GB 35380 from the National Science Foundation and by U.S. Office of Naval Research contract N-00014-67-A-0226-0003. Dr M.J. Readhead of the Pharmaceutical Division, Reckitt \& Colman, Ltd, Hull, England, kindly supplied the RX72601. G.M. was visiting Assistant Professor in Psychobiology from Hull, England, and is grateful to the Wellcome Trustees for their award of a travel grant. P.C.R. was a David Ross pre-doctoral fellow of the Purdue Research Foundation. Reprint requests to R.D.M. 


\section{References}

AVERY, D.D. (1970). Hyperthermia induced by direct injections of carbachol in the anterior hypothalamus. Neuropharmac., 9, 175-178.

CONNOR, J.D., ROSSI, G.V. \& BAKER, W.W. (1966). Analysis of the tremor induced by injection of cholinergic agents into the caudate nucleus. Int. $J$. Neuropharmac., 5, 207-216.

DETTMAR, P.W., LEWIS, J.W., METCALF, G., READHEAD, M.J., SMITH, M.H., GILLETT, G.B., HEDGES, A. \& RICHENS, A.R. (1974a). New inhibitor of acetylcholinesterase with preferential activity at nicotinic sites. J. Pharm. Pharmac., 26, 134-135.

DETTMAR, P.W., METCALF, G., SMITH, M.H., GILLETT, G.B., HEDGES, A. \& RICHENS, A. (1974b). Preliminary assessment of RX72601, a new anticholinesterase in man: Reversal of competitive neuromuscular blockade. Br. J. clin. Pharmac., 1, 217-222.

FELDBERG, W., MYERS, R.D. \& VEALE, W.L. (1970). Perfusion from cerebral ventricle to cisterna magna in the unanaesthetized cat. Effect of calcium on body temperature. J. Physiol., 207, 403-416.

FELDBERG, W. \& SAXENA, P.N. (1970). Mechanism of action of pyrogen. J. Physiol., 211, 245-261.

HALL, G.H. (1972). Changes in body temperature produced by cholinomimetic substances injected into the cerebral ventricles of unanaesthetized cats. $B r . J$. Pharmac., 44, 634-641.

HALL, G.H. \& MYERS, R.D. (1971). Hypothermia produced by nicotine perfused through the cerebral ventricles of the unanaesthetized monkey. Neuropharmac., 10, 391-398.

HALL, G.H. \& MYERS, R.D. (1972). Temperature changes produced by nicotine injected into the hypothalamus of the conscious monkey. Brain Res., 37, 241-251.

LEWIS, J.W., READHEAD, M.J., METCALF, G., SMITH, M.H. \& McNALLY, P.H. (1973). Cis-N-2-arylcyclohexylamines and their quaternary ammonium salts-two new series of cholinesterase inhibitors. $J$. Pharm. Pharmac., 25, 152-153.

METCALF, G. \& DETTMAR, P.W. (1975). Evaluation of
RX72601 as an anticurare agent. Br. J. Anaesths., 47, $451-456$.

MYERS, R.D. (1964). Emotional and autonomic responses following hypothalamic chemical stimulation. Canad. J. Psychol., 18, 6-14.

MYERS, R.D. (1970). An improved push-pull cannula system for perfusing an isolated region of the brain. Physiol. Behav., 5, 243-246.

MYERS, R.D. (1971a). Primates. In: Comparative Physiology of Thermoregulation, ed. Whittow, C.C. Vol. 2, pp. 283-326. New York: Academic Press.

MYERS, R.D. (1971b). General laboratory procedures. In: Methods in Psychobiology, ed. Myers, R.D. Vol. I, pp. 27-65. London: Academic Press.

MYERS, R.D. (1972). Methods for perfusing different structures of the brain. In: Methods in Psychobiology, ed. Myers, R.D. Vol. II, pp. 169-211. London: Academic Press.

MYERS, R.D. (1974). Handbook of Drug and Chemical Stimulation of the Brain. New York: Van Nostrand Reinhold Company.

MYERS, R.D. \& WALLER, M.B. (1973). Differential release of acetylcholine from the hypothalamus and mesencephalon of the monkey during thermoregulation. J. Physiol., 230, 273-293.

MYERS, R.D. \& YAKSH, T.L. (1969). Control of body temperature in the unanaesthetized monkey by cholinergic and aminergic systems in the hypothalamus. J. Physiol., 202, 483-500.

NORTON, S. (1969). The effects of psychoactive drugs on cat behaviour. Ann. N.Y. Acad. Sci., 159, 915-927.

RUDY, T.A. \& WOLF, H.H. (1972). Effect of intracerebral injections of carbamylcholine and acetylcholine on temperature regulation in the cat. Brain Res., 38, 117-130.

WOLF, G. (1971). Elementary histology for neuropsychologists. In: Methods in Psychobiology, ed. Myers, R.D. Vol. I, pp. 281-300. London: Academic Press. 